\title{
Japan plans new lunar mission
}

\section{Tokyo}

SCIENTISTs at Japan's Institute of Space and Astronautical Science (ISAS) are planning an ambitious follow-up to their recent success in sending a spacecraft to the Moon (see Nature 343, 403; 1 February 1990). Last week, a committee at the institute decided to give priority to a mission to monitor 'moonquakes' by dropping penetrators on the Moon from an orbiting spacecraft.

The institute's next lunar mission is tentatively scheduled for 1995 and will use the new larger $\mathrm{M}-\mathrm{V}$ solid-fuel rocket currently under development by the institute. It will be capable of carrying much larger payloads than its present launch vehicle, the M-3SII.

The spacecraft will drop three $13-\mathrm{kg}$, $12-\mathrm{cm}$ diameter penetrators, each about the length of a cricket bat, from orbit onto widely spaced locations on the lunar surface: one near a former Apollo landing site, one on the far side of the Moon, and another near the northern lunar pole. Retro-motors fitted in the penetrators will reduce the speed of impact, but it will still be a 'hard landing' at a speed of 250-300 metres per second, according to Hitoshi Mizutani of ISAS.

Mizutani says the penetrators will plunge 1-3 metres into the lunar regolith (topsoil). Tiny seismometers and other instruments carried in the penetrators will then relay information on 'moonquakes' and heat flow to Earth via the orbiting mother spacecraft. The instruments are expected to keep working for about one year.

ISAS scientists have been perfecting the design of the penetrators by firing lifesized models into artificial lunar soil with a high-speed gun at their Noshiro Rocket Center in northern Japan. Details of the technology developed for protecting the sensitive seismometers from lunar impact are "secret", says Mizutani. But basically the instruments will be packed in light epoxy material and great care will be taken to ensure that there are no moving parts that could be displaced as the penetrators hit the Moon at close to the speed of sound.

The plan has to cross several hurdles before it wins approval and funding from the Ministry of Education, Science and Culture (MESC), to which ISAS is affiliated. According to Jun Nishimura, director general of ISAS, the project will be screened by another couple of committees before official ISAS approval can be granted in June. It will then be for MESC to decide if funding will be requested from the Ministry of Finance.

The lunar project was one of three being considered by ISAS for launch in the mid-1990s. The others are a mission to drop a balloon into the atmosphere of Venus and an ambitious proposal to collect dust from the comet Wirtanen and return it to Earth. The lunar mission is being given priority and the others will be taken up for discussion next year.

David Swinbanks

\section{Astronomers' wait continues}

\section{Greenbelt, Maryland}

A sticking valve in the Space Shuttle's hydraulic system stopped the long-delayed launch of the Hubble space telescope four minutes before its scheduled take off last week. Replacing a key part is expected to take one to two weeks, with the next launch attempt scheduled for 25 April, the National Aeronautic and Space Administration (NASA) said.

The shuttle and its $\$ 2,000$ million payload will remain on the launch pad at Kennedy Space Center during the repairs. NASA's main worry now is that the telescope could be contaminated as technicians work on the shuttle and repair the telescope's batteries. Air pressure within the shuttle's cargo bay is maintained at higher than atmospheric level to keep dust and other contaminants out.

But to recharge the batteries, technicians will need to enter the compartment, which is normally maintained at near clean-room standards to prevent the entry of dirt or even insects that could show up as specks in the telescope's sensitive optics. The extra two weeks on the ground is nevertheless expected to increase contamination of the Hubble's mirror by about 0.1 per cent

NASA officials say they will surround the cargo bay with a form-fitting shell during the repairs. Although air pressure within the bay will drop as the bay doors are opened, NASA expects that the airtight gantry will keep pressure in the cargo area above atmospheric levels, and thus relatively free of contaminants.

The faulty valve is part of the first auxiliary power unit, one of three hydrazine-powered engines that run the turbines that maintain the shuttle's hydraulic pressure. When NASA technicians install a replacement the power unit this week, it will be the first time that such a procedure has been attempted on the launch pad, with the shuttle in a vertical position. Because the operation is unfamiliar, NASA is allowing extra time for its completion.

G. Christopher Anderson

\section{SERC's saving is Scotland's loss}

\section{London}

AN Anglo-German review panel has given preliminary approval to a plan to build a gravity-wave observatory, which could be completed as early as the mid-1990s. But to the disappointment of the British teams involved in the project, the observatory seems likely to be built in West Germany, rather than in Britain. The project is expected to begin after formal approval is granted by the UK Science and Engineering Research Council (SERC) in July.

British physicists hoped that the observatory would be built in Tentsmuir forest, Fife, in Scotland. Although the site is excellent, West Germany will have the right to choose the observatory's location as SERC plans to contribute only about $£ 5$ million towards the project's estimated $£ 30$ million costs. German funding is expected to come from the Research Ministry (BMFT), the local Land and the Max Planck Society.

Professor Jim Hough, from the University of Glasgow, says that his group (which designed the observatory together with a German team from the Max Planck Institute for Quantum Optics at Garching) had hoped that SERC would pay for a large part of the project, but were told informally about a year ago that most of the money would have to come from abroad.

Hough still hopes the detector can be built in Scotland, should any problems arise in Germany. The first German site to be selected, in Bavaria, was abandoned because of difficulties in gaining planning permission. Although approval for a site just north of Hanover should be easier, German physicists are still waiting for the final word from the local authority. Hough's team has obtained planning permission for the Tentsmuir site.

The observatory will consist of four large suspended masses, at the end of two 3-kmlong arms, arranged in an ' $L$ ' shape. The interference patterns of laser light bouncing between mirrors on the masses will show up extremely small changes in their spacing - changes that could be caused by ripples in space-time as predicted by the theory of general relativity.

These ripples, or 'gravity waves', should arise from the movement of enormous masses - for example the collapse of a star's core in a supernova explosion. Observing these gravitational waves has been described as "one of the last-known windows that remains to be opened on the Universe" (see Nature 321, 378; 1986).

The US National Science Foundation also intends to build gravity-wave detectors and has plans for a pair of 4-km arm facilities to be located at still-undecided sites in the eastern and western United States. If Congress agrees to fund the project this year then the detectors should be in operation by $1995 . \quad$ Peter Aldhous 